\title{
Highly efficient Q-switched Ho:YLF laser pumped by Tm:fiber laser
}

\author{
Jirong $\mathrm{Yu}^{2}$, Yingxin Bai ${ }^{1}$, M. Petros ${ }^{3}$, Paul Petzar ${ }^{4}$, Bo Trieu ${ }^{2}$, Hyung Lee ${ }^{4}$, U. Singh ${ }^{2}$ \\ ${ }^{1}$ Science Systems and Applications, Inc, One Enterprise Parkway, Hampton, VA 23666 \\ Y.bai@larc.nasa.gov \\ 2NASA Langley Research Center, Hampton, VA 23681 \\ ${ }^{3}$ Science and Technology Corporation, 101 Research Drive, Hampton, VA 23666 \\ 4 National Institute of Aerospace , 100 Exploration Way, Hampton , VA 23668
}

\begin{abstract}
A highly efficient Q-switched Ho:YLF laser pumped by a Tm:fiber laser has been designed and demonstrated. When the pump power is $30 \mathrm{~W}$, the pulse energy is $30 \mathrm{~mJ}$ at the repetition rate of $100 \mathrm{~Hz}$.
\end{abstract}

OCIS codes: (140.3580) Lasers, solid-state; (140.3510) Lasers, fiber.

\section{Introduction}

There are many specific applications for $2 \mu \mathrm{m}$ lasers in atmospheric surveillance and medical therapy such as wind/ $\mathrm{CO}_{2}$ measurement and hard and soft tissue ablation. The salient features of $2 \mu \mathrm{m}$ lasers are eyesafety, high water absorption, and strong aerosol scattering [1]. Currently a diode pumped Tm:Ho:LuLiF system, which consists of a master Q-switched oscillator and three power amplifiers, has been developed at NASA Langley Research Center (LaRC). The output pulse energy is more than $1 \mathrm{~J}$ [2]. The dipole-dipole conversion in a Tm:Ho:co-doped laser crystal can convert the pump energy of diodes to the population inversion of Ho. The drawback of Tm:Ho co-doped laser is low efficiency because of the upper state absorption, Tm and Ho energy sharing, concentration quenching, and large heat loading. For the air and space borne lidar applications, it is very important to enhance efficiency of $2 \mu \mathrm{m}$ lasers. Theoretically, doping Ho and Tm in two separate crystals is helpful to eliminate or alleviate the mentioned-above effects. Experimentally, higher efficiency has been obtained from a diode pumped Tm:YAG laser pumped Ho:YLF laser [3]. With the advancement of Tm fiber lasers, continuos-wave output power of more than 100W is commercially available. This Tm fiber laser can be used to pump Ho lasers. Ho doped lasers have a larger effective stimulated-emission cross-section and lower saturation fluence [4]. Both large effective stimulated-emission cross-section and low saturation fluence are good for energy extraction of Q-switched oscillator or pulsed amplifier. Previously, we have reported the high efficiency operation of a Tm:fiber laser pumped Ho:YLF laser in continuous-wave mode [5]. In this paper we report the highly efficient operation of a Tm fiber laser pumped Ho:YLF laser in Q-switching mode.

\section{The optimal design of Q-switched Ho:YLF laser}

In continuous-wave operation, a small pump beam size without damaging the crystal is more efficient; however, Q-switch operation of a Tm:fiber pumped Ho:YLF laser requires a large energy storage. The storage of pump energy depends on the pump intensity, pump beam size, crystal length, and repetition rate. Fig.1 shows the analytical result based on a developed model at LaRC. With limited power, if the pump volume in the laser crystal is too large, the average pump intensity will be lower leaving most of pump power depleted to make population inversion. When the pump volume is too small, it is possible to damage the crystal. Even though the crystal is not damaged at small pump volume, the strong pump density results in the saturation of pump absorption and affects the laser efficiency. The expected pulse repetition rate for the system is $100 \mathrm{~Hz}$ and the calculated optimal pump intensity is around $2 \mathrm{~kW} / \mathrm{cm}^{2}$. 


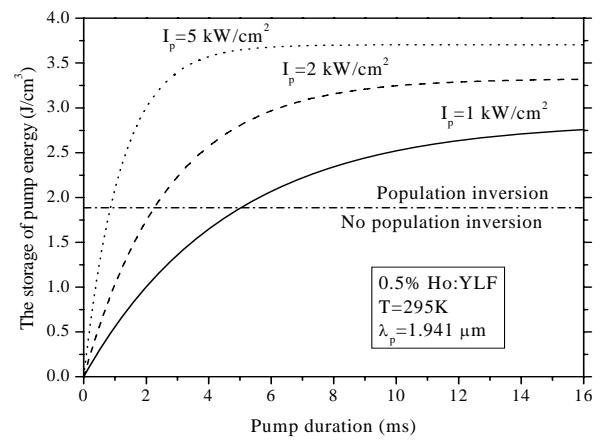

Fig.1 The storage of pump energy versus pump duration

\section{The operation of Q-switched Ho:YLF laser}

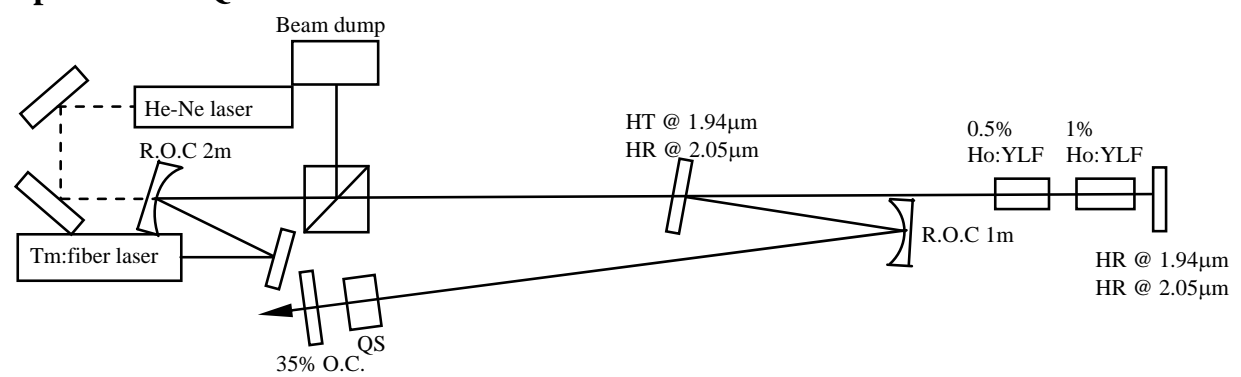

Fig.2 The Q-switched Ho:YLF laser pumped by a Tm:fiber laser.

Fig.2 shows the configuration of the designed Q-switched Ho:YLF laser which is pumped by a Tm:fiber laser. For the mode matching, a folded configuration is used where both pump and laser beams have the same confocal parameters. The maximum output power of Tm:fiber pump laser is $40 \mathrm{~W}$ in random polarization. The center wavelength of Tm:fiber laser is $1.941 \mu \mathrm{m}$, a difference of $2 \mathrm{~nm}$ from the absorption peak of Ho:YLF crystal. The output of the Tm: fiber laser beam is $5 \mathrm{~mm}$ in diameter. In order to make the pump intensity in the crystal above $2 \mathrm{~kW} / \mathrm{cm}^{2}$, This pump beam is focused on the Ho:YLF crystal by a concave mirror with $2 \mathrm{~m}$ radius of curvature. The radius of the focused beam is $0.55 \mathrm{~mm}$ at waist and the full divergence angle of the focused beam is $2.17 \mathrm{mrad}$. By appropriate feedback from the back mirror of the laser, the polarization of Tm:fiber laser is changed from random to linear polarization. This results in $>75 \%$ pump power (30W) incident on the Ho:YLF crystal along $\pi$ polarization. The first pumped laser crystal is $0.5 \%$ Ho:YLF and its dimension is $5 \mathrm{~mm} \times 5 \mathrm{~mm} \times 25 \mathrm{~mm}$. The second pumped laser crystal is $1 \%$ Ho:YLF crystal and its dimension is $5 \mathrm{~mm} \times 5 \mathrm{~mm} \times 30 \mathrm{~mm}$. Both crystals are cooled by water at $10^{\circ} \mathrm{C}$. The laser cavity consists of two flat highly reflective mirrors, one $1 \mathrm{~m}$ radius concave and one flat output coupler with $35 \%$ reflectivity. The total laser cavity length is $1.65 \mathrm{~m}$.

30mJ of output pulse energy from this Q-switched Ho:YLF laser pumped by a Tm:fiber laser is obtained at a repetition rate of $100 \mathrm{~Hz}$. The corresponding optical to optical efficiency is $10 \%$ which is double than that of Ho:Tm co-doped lasers. Further improvement and be realized by pumping the Ho:YLF crystals from both sides to fully utilize the pump power.

\section{References:}

1. Mulugeta Petros, Jirong Yu, Tony Melak, Bo Trieu, Songsheng Chen, Upendra N. Singh, and Yingxin Bai, "High energy totally conductive cooled, diode pumped, $2 \mu \mathrm{m}$ laser”, in ASSP 2005 OSA Topical Meeting, MB11.

2. Jirong Yu, Bo C. Trieu, Ed A. Modlin, Upendra N. Singh, Micheal J. Kavaya, Songsheng Chen, Yingxin Bai, Paul J. Petzar, and Mulugeta Petros, “1 J/pulse Q-switched 2- $\mu$ m solid-state laser”, Opt. Lett. Vol.31 462-464 (2006)

3. Alex Dergachev, Peter F. Moulton, and T. E. Drake, "High-power, High-Energy Diode Pumped Tm:YLF-Ho:YLF-ZGP laser system”, OSA TOPS Vol.83, John J. Zayhowski ed. 137-141,2003.

4. Norman P. Barnes, Waldo J. Rodriguez, Brian M. Walsh, “Ho:Tm:YLF laser amplifiers”, J. Opt. Soc. Am. B, Vol.13, 28722882 (1996).

5. Yingxin Bai, M. Petros, Jirong Yu, Paul Petzar, Bo Trieu, Hyung Lee, U. Singh, "Highly efficient Ho:YLF laser pumped by Tm:fiber laser", in CLEO/QELS and Phast 2006, (Long Beach, California) CThFF 4. 